\title{
Educação e Rendimentos dos Ricos no Brasil
}

\author{
Marcelo Medeiros ${ }^{1}$ \\ Juliana de Castro Galvão \\ ${ }^{1}$ Universidade de Brasília (UnB), Brasília, DF, Brasil. E-mail: marcelo.medeiros.cs@gmail.com
}

\section{INTRODUÇÃO}

objetivo deste artigo é avaliar em que medida a educação pode ser considerada um dos principais determinantes da riqueza no Brasil. Definimos como ricas as pessoas no $1 \%$ mais rico da distribuição de rendimentos do trabalho. Focamos tanto na educação universitária em geral quanto nos cursos de elite, os quais são definidos como aqueles em que as chances de encontrar alguém acima da linha de riqueza são muito superiores às de outros cursos. Isso ocorre, por exemplo, com os cursos de medicina ou engenharia. Nosso interesse é na associação entre educação e riqueza, tanto no plano individual quanto no agregado, ou seja, queremos examinar como a educação aumenta as chances de um indivíduo pertencer ao $1 \%$ mais rico da distribuição dos rendimentos do trabalho e como os efeitos da educação universitária geral e de elite afetam os níveis totais de riqueza na população.

Os ricos são um grupo-chave no estudo da estratificação social. As razões para estudá-los são quase autoevidentes, uma vez que eles se apropriam de uma fração desproporcional da renda de uma sociedade. Além disso, o controle dos ricos sobre recursos econômicos tende a influir também em outros domínios da vida, tais como a política e a cultura.

DADOS - Revista de Ciências Sociais, Rio de Janeiro, vol. 59, n-2, 2016, pp. 357 a 383. 
Há razões para dar atenção aos rendimentos do trabalho quando se estudam os ricos. Em diversos países aqueles no 1\% superior da distribuição de todos os rendimentos são os ditos "ricos trabalhadores", cuja principal fonte de renda é a remuneração do trabalho e não a do capital (Bakija, Cole e Heim, 2010; Fortin et al., 2012; Godechot, 2012; Kaplan e Rauh, 2010; Keister, 2014; Keister e Lee, 2014; Parker e Vissing-Jorgensen, 2010; Saez e Veall, 2005; Sanhueza e Mayer, 2011; Wolff, 2000). De fato, dados tributários indicam que os rendimentos do trabalho são o principal componente da renda do $1 \%$ mais rico na distribuição de renda de todas as fontes na Colômbia (Vélez, 2012:23), Argentina (Alvaredo, 2010), Indonésia (Leigh e Van der Eng, 2009), França (Landais, 2008), Canadá (Fortin et al., 2012) e Estados Unidos (Feenberg e Poterba, 2000; Piketty e Saez, 2013). Ao que parece o Brasil não é uma exceção, como sugerem os dados de pesquisas domiciliares (Albuquerque, 1994; Medeiros, 2005).

O papel que a educação tem como determinante da riqueza merece atenção por pelo menos duas razões. Primeiro, devido aos limites que as políticas educacionais podem apresentar se usadas como ferramenta de redução da desigualdade - se a educação não for relevante para os ricos, então haverá uma parte importante da desigualdade total que não será afetada por políticas educacionais. Segundo, devido à associação entre educação e meritocracia, e a importância dessa associação para o discurso político justificando os altos níveis de concentração da renda nos ricos.

Para conduzir o estudo utilizamos dados de uma pesquisa de grande escala, a Amostra do Censo Demográfico de 2010, do Instituto Brasileiro de Geografia e Estatística (IBGE) que coleta informações sobre rendimentos do trabalho, características demográficas dos trabalhadores e sua formação específica (tipo de diploma de nível superior), quando for o caso. Há razões para crer que esse tipo de dado atende às demandas de nossa análise além dos rendimentos do trabalho serem menos propensos a subdeclaração que outros tipos de rendimento, a grande amostra de nossa pesquisa diminui a ocorrência de problemas normalmente associados ao estudo dos ricos a partir de levantamentos amostrais, assunto que já foi objeto de discussão por Saez (2006), Atkinson (2007), Atkinson, Piketty e Saez (2011) e, no Brasil, por Medeiros, Souza e Castro (2015a, 2015b).

Com a finalidade de examinar os efeitos da educação sobre os rendimentos, estimamos equações de salários usando regressões lineares; 
para avaliar seu impacto nos níveis de riqueza, fazemos simulações contrafactuais de quais seriam as distribuições de rendimentos sem esses efeitos. Nossa principal conclusão é que a educação de elite aumenta, sim, as chances de um indivíduo pertencer ao 1\% mais rico, porém não há evidência de que a educação geral seja um dos principais fatores explicando a riqueza no Brasil.

\section{ESTUDOS ANTERIORES}

A pesquisa relacionando educação e riqueza tem início na sociologia e remonta pelo menos ao trabalho de Sorokin (1925), que estudou os milionários nos Estados Unidos e mostrou que, embora eles possuíssem níveis educacionais superiores aos do restante da população, esses níveis mais altos não pareciam suficientes para justificar uma riqueza muito maior. Isto, no entanto, referia-se a um grupo extremamente rico e poderia simplesmente ser um caso excepcional. Além disso, a maior parte dos ultrarricos estudados por Sorokin viveu no século XIX, quando educação, renda e riqueza não possuíam a mesma associação que têm hoje.

A literatura mais recente, porém, mantém as conclusões obtidas há mais de um século. Inhaber e Carroll (1992), por exemplo, afirmam que anos de estudo não são um bom preditor de rendimentos na parte superior da distribuição. Seu argumento é que a teoria do capital humano pode ser razoável para prever rendimentos de $90 \%$, talvez $95 \%$, da população, mas não parece adequada para explicar o que acontece com os mais ricos. Ainda que os ricos possam ser mais ricos porque possuem talentos especiais, não existe nenhuma evidência de que esses talentos tenham sido adquiridos no sistema educacional.

A verdade é que as conclusões de Sorokin e de Inhaber e Carroll encontram apoio em um corpo muito maior da literatura sobre mobilidade intergeracional que indica que o papel da educação formal na mobilidade varia conforme as classes de origem, e tende a ser menos importante para aqueles cujos pais vêm de classes mais altas. Heranças de patrimônio, por exemplo, têm um papel importante na transmissão de vantagens entre gerações para as classes mais altas (Broom e Shay, 2000; Canterbery e Nosari, 1985; Hurd e Mundaca, 1989; Keister e Lee, 2014; Lundberg, 1969; Russell, 1979; Stilwell e Jordan, 2007; Tickamyer, 1981; Wolff, 2002). Além disso, a transmissão não ocorre apenas entre gerações, mas, também, lateralmente, isto é, dentro de uma mesma 
geração. Laços familiares colaterais aumentam expressivamente as chances de um indivíduo ter patrimônio elevado (Broom e Shay, 2000).

Há razões para crer que, além da transferência de patrimônio, há outras formas de transmissão de vantagens. Elas incluem a mobilização de redes sociais, ter famílias que permitam assumir maiores riscos, bem como outros fatores que influenciam a forma como os indivíduos participam da economia. Todavia, é muito difícil medir isso diretamente, e a maior parte da literatura que aponta nessa direção o faz por exclusão das explicações alternativas (Breen e Jonsson, 2005; Erikson e Goldthorpe, 2002; Finnie e Irvine, 2006; Piketty, 2000; Spilerman, 2000; Torche, 2014; Torche e Costa-Ribeiro, 2012; Torche e Spilerman, 2008, 2009). O que talvez seja mais importante no nosso caso é que uma dessas explicações alternativas excluídas frequentemente é a mobilidade educacional, o que indica que ter níveis elevados de educação formal sem ter as outras vantagens mencionadas pode impor um limite para o posicionamento no topo da hierarquia social.

Estudos voltados especificamente para a questão de como a educação é capaz de explicar altos rendimentos no Brasil são limitados em quantidade. Examinando dados sobre os ricos brasileiros, Albuquerque (1994) e Ferreira (2001) encontraram elevados níveis de escolaridade entre os ricos, embora não tenham avaliado em que medida esse era um fator que os diferenciasse do restante da população. Medeiros (2005), porém, realiza um estudo com esse objetivo, e conclui que as credenciais educacionais não são suficientes para explicar por que algumas famílias são ricas: enquanto educação parece ser um fator importante para explicar a pobreza, não há evidências de que seja capaz de predizer a riqueza. Usando simulações contrafactuais, Medeiros argumenta que as diferenças de escolaridade não são capazes de responder por esse tipo de desigualdade: atribuir a todos os trabalhadores do país o efeito sobre os rendimentos de um diploma universitário moveria não mais que $1 \%$ das famílias para a classe dos ricos (os $0,9 \%$ mais ricos, definidos por uma linha de riqueza). Seu argumento é que se a educação for capaz de explicar a riqueza, a desigualdade entre os ricos e o resto deve resultar de diferenças no tipo de educação. Esta última ideia é a que pretendemos examinar em detalhe.

Nosso estudo apresenta algumas vantagens em relação a Medeiros (2005). Primeiro, somos capazes de analisar com muito mais refinamento o tipo de educação superior dos indivíduos, pois dispomos dos 
títulos dos cursos concluídos, enquanto Medeiros trata apenas de uma ampla categoria "com nível superior". Temos, portanto, detalhamento da categoria educacional que apresenta maior heterogeneidade interna de renda. Segundo, Medeiros usa dados das Pesquisas Nacionais por Amostra de Domicílios (PNADs) da década de 1990, os quais subestimam a renda no topo da distribuição quando comparados a todas as outras pesquisas domiciliares nacionais (Souza, 2015), ao passo que aqui usamos dados do Censo, que tendem menos à subestimação.

\section{METODOLOGIA}

Nossos dados provêm do Questionário da Amostra do Censo Demográfico 2010, ao qual, por simplificação, nos referiremos como Censo 2010. O tamanho dessa amostra é de aproximadamente 20,6 milhões de registros (indivíduos), representando uma população de 190,7 milhões de pessoas. O grande tamanho possivelmente reduz deficiências de amostragem na coleta de informação sobre rendimentos do trabalho dos ricos. Como os rendimentos no Censo, na prática, não são limitados, e como antes da divulgação dos dados é feita uma série de testes para identificar e corrigir erros de preenchimento, há razões para crer que, para nossos propósitos específicos, a base de dados reflete aceitavelmente o extremo superior da distribuição dos rendimentos do trabalho, embora muito provavelmente estes estejam subestimados acima do quantil do $1 \%$ mais rico. A subpopulação estudada é aquela de trabalhadores adultos com idades entre 25 e 64 anos, cujos rendimentos eram positivos. Ao processar os dados excluímos todos os registros com valores ignorados em qualquer uma das variáveis de nossa subamostra (uma exclusão de $0,6 \%$ da subpopulação de trabalhadores adultos, sem padrão claro observável). A subamostra final tem um tamanho de 6,4 milhões de registros, os quais representam um grupo de 62,9 milhões de pessoas.

Em termos gerais, nossa abordagem segue quatro etapas. Na primeira, para descrever a composição educacional da população por classe e calcular as chances relativas de estar em cada classe, por nível educacional, ordenamos os trabalhadores em função dos rendimentos de todos os trabalhos e dividimos essas pessoas em duas classes, uma dos quantis $0 \%$ a $99 \%$ e outra do $99 \%$ ao $100 \%$ (topo $1 \%$ ). Na segunda, calculamos o efeito líquido de cada nível de educação nas chances de pertencer ao 1\% mais rico usando regressões logísticas. Na seguinte, por meio da regressão linear de uma equação de salários obtivemos os 
parâmetros de diferentes grupos educacionais para assim fazer simulações de rendimentos assumindo reduções nos retornos de distintas categorias educacionais, o que na prática equivale a simular mudanças no nível educacional da população. Na quarta etapa calculamos os impactos das simulações anteriores usando as medidas de riqueza discutidas por Peichl, Schaefer e Scheicher (2010).

Nossa unidade de análise são trabalhadores, não postos de trabalho. Definimos como ricos o $1 \%$ mais rico na distribuição de todos os rendimentos brutos do trabalho dos adultos. Como linha de riqueza usamos o nível de rendimento do percentil 99\%, R\$ 11.000, valores de julho de 2010. Nas regressões, linear MQO e logística MVS, todos os modelos seguem a mesma especificação geral, exceto no que se refere às variáveis dependentes e às variáveis educacionais. Um grupo de regressões usa categorias educacionais amplas (primário, secundário e universitário) enquanto outro subdivide o nível universitário em categorias específicas de treinamento, como se discute adiante.

Para as regressões lineares especificamos uma equação de salários, com a variável dependente sendo o logaritmo natural dos rendimentos mensais do trabalho. Nas regressões logísticas as categorias dependentes são: pertence ou não pertence ao estrato $1 \%$ mais rico (ou seja, está ou não acima da linha de riqueza), com o restante da especificação idêntica à das equações de salários, exceto pelo quadrado da idade. As variáveis independentes estão listadas a seguir. As bases das variáveis dummies são a primeira categoria listada em cada variável:

- Idade: um polinômio formado pela idade em anos e o quadrado da idade; na regressão logística, idade em anos, mas não o quadrado da idade - esta é a única diferença entre as regressões logística e linear nas variáveis independentes.

- Sexo: feminino, masculino.

- Região de residência: as cinco macrorregiões geopolíticas do IBGE, base Nordeste.

- Cor: negros, incluindo pretos, pardos e indígenas e brancos, agregando brancos e asiáticos.

- Educação geral: menos que educação primária completa (igual ou menos que 8 anos de estudo), primário completo porém secundário incompleto (mais que 8 e menos que 12 anos), secundário completo po- 
rém universitário incompleto (de 12 a 15 anos), universitário completo, mestrado e doutorado. O treinamento específico merece comentários adicionais.

O Censo 2010 classifica informação sobre treinamento universitário de acordo com os Fields of Education and Training da Eurostat, o qual, por sua vez, reflete o conteúdo do International Standard Classification of Education 1997 (ISCED-97). Há uma forte conexão entre a ISCED e a International Standard Classification of Occupations 1988 (ISCO-88). Deste modo, as classificações de treinamento usadas no Censo são em grande medida comparáveis com outras classificações educacionais no mundo e, apesar da diferença nas dimensões (educação versus ocupação), são também comparáveis com classificações baseadas em títulos ocupacionais, tais como a maioria dos esquemas de classe utilizados no estudo da mobilidade social.

Para o treinamento específico, partimos das classificações do Censo e agrupamos os diplomas universitários em 11 categorias (códigos ISCED-97 / Censo em colchetes): 1. Formação de Professores e Ciências da Educação [140-46]; 2. Humanidades, Psicologia, Ciências Sociais, Serviço Social e Artes [210-15, 220-26, 310-313, 761-762]; 3. Administração, Negócios e Economia [314, 340-47]; 4. Jornalismo e Informação [321-22]; 5. Direito [380]; 6. Matemática, Física, Estatística e Ciências da Vida [420-22, 440-43, 461-62]; 7. Engenharia, Ciência da Computação, Ciências da Produção, Construção e Arquitetura [481-83, 520-25, 581-82]; 8. Agricultura, Veterinária, Alimentos, Extração e Mineração [540-44, 620-24, 641]; 9. Saúde (exclui Medicina) [720, 723-27]; 10. Medicina [721]; 11. Serviços Pessoais, Transporte, Proteção Ambiental e Defesa [810-15, 840, 850-53, 860-63].

A ordem das categorias de formação específica reflete, em larga medida, as habilidades necessárias para executar atividades ISCO-88. Por essa razão, agrupamos as categorias de formação usando dois critérios de agregação: i) caso elas pertencessem a categorias vizinhas no ISCED-97 e ii) caso as pessoas nas categorias possuíssem razões de chances de pertencer ao $1 \%$ mais rico da distribuição de rendimentos de todos os trabalhos que fossem similares, mesmo quando controlando por características pessoais por meio de regressões logísticas em testes prévios. Ao proceder desta maneira, tentamos agrupar habilidades semelhantes sem introduzir demasiada heterogeneidade intragrupo. Uma categoria agregou todos os mestrados e outra todos os douto- 
rados, independente da área de conclusão da pós-graduação. Todas essas categorias entraram nas regressões como dummies, tendo como base menos que o primário completo.

A estimativa dos efeitos da formação geral e específica sobre os níveis de riqueza foi realizada por meio de simulações contrafactuais. O procedimento simula quais seriam a distribuição de renda e os níveis de riqueza caso a vantagem líquida de, por exemplo, a educação superior, fosse suprimida dos rendimentos de cada indivíduo. O ponto de partida das simulações são as regressões lineares descritas anteriormente. Os resultados dessas regressões encontram-se no Apêndice 1. A regressão estima os parâmetros de cada variável - incluindo as variáveis educacionais -, prevê os logaritmos naturais dos rendimentos do trabalho e calcula os resíduos dessa predição. Nós assumimos, como se faz regularmente, que os parâmetros estimados correspondem ao efeito líquido das variáveis nos logaritmos naturais dos rendimentos do trabalho. O passo seguinte foi suprimir a vantagem líquida da educação universitária por meio da substituição dos parâmetros estimados para uma formação universitária pelos parâmetros estimados para uma formação inferior - educação secundária - e recalculando os rendimentos por simulação para gerar uma nova distribuição de renda e novas medidas de riqueza, dada uma linha de riqueza. Na prática, isso é o equivalente a simular a imposição de um teto na educação de forma que ninguém tenha mais do que educação secundária. Repetimos o procedimento simulando um teto no nível de alguma formação específica, tais como Administração, Negócios e Economia ou, ainda, Formação de Professores e Ciências da Educação.

Nossas simulações são parciais e estáticas. Evidentemente, não podemos esperar precisão desse tipo de simulações, uma vez que os rendimentos dos trabalhadores são interdependentes. Uma redução nos rendimentos dos trabalhadores no topo da distribuição pode, por exemplo, implicar uma redução em seu consumo e, consequentemente, na diminuição dos salários dos trabalhadores que provêm bens e serviços a eles. Ou, na direção oposta, poderia aumentar o uso de trabalho qualificado na economia, aumentar a competitividade internacional e fazer a economia crescer no longo prazo. Calcular precisamente o que aconteceria em um mercado de trabalho dinâmico está além de nossa capacidade e completamente fora de nossos propósitos. O objetivo de nossas simulações é produzir um indicador da importância da educação para cada indivíduo no topo da distribuição e não prever 
como mudanças educacionais agregadas modificariam o mercado de trabalho.

Simulações estáticas são, indiscutivelmente, um ponto fraco de nossa metodologia. Por essa razão evitamos discutir e interpretar as magnitudes específicas das mudanças nas medidas de riqueza decorrentes das simulações. Precisão, neste caso, deixa de ser importante. Todavia, não há razões para crer que os problemas associados às simulações contrafactuais possam afetar nossas conclusões substantivas a respeito da educação para os ricos.

\section{RESULTADOS}

\section{Composição da Força de Trabalho Adulta}

O trabalhador brasileiro típico tem níveis educacionais baixos. Mais da metade da força de trabalho adulta sequer possui educação secundária completa. Apenas um sexto tem diploma universitário, incluindo-se pessoas com mestrado e doutorado, com as duas últimas categorias somando apenas $1 \%$ da força de trabalho, como mostra a Tabela 1. É provável que diferenças na qualidade da educação amplifiquem a desigualdade nas quantidades de educação.

Essa composição é completamente invertida entre o $1 \%$ mais rico dos trabalhadores, com $76,8 \%$ possuindo um diploma universitário, dos quais $9,3 \%$ e $5,1 \%$ possuem, respectivamente, mestrado e doutorado. Diante da forte correlação entre educação e rendimentos no Brasil, este é um resultado esperado. É também possível encontrar 14,1\% dos trabalhadores com educação secundária completa entre o $1 \%$ mais rico, dos quais alguns estão cursando universidade e obterão um diploma em poucos anos.

Ter elevado nível de escolaridade é uma característica compartilhada dentro do 1\% mais rico. A questão relevante, porém, é em que medida um diploma universitário é suficiente para colocar alguém no 1\% mais rico. Para examinar isso dividiremos o nível universitário em categorias e examinaremos as chances relativas de pertencer ao topo da distribuição (estar acima da linha de riqueza) dos trabalhadores em cada categoria educacional.

Quatro categorias de cursos de graduação dominam o 1\% mais rico, representando mais de dois terços da classe: i) Administração, Negócios 


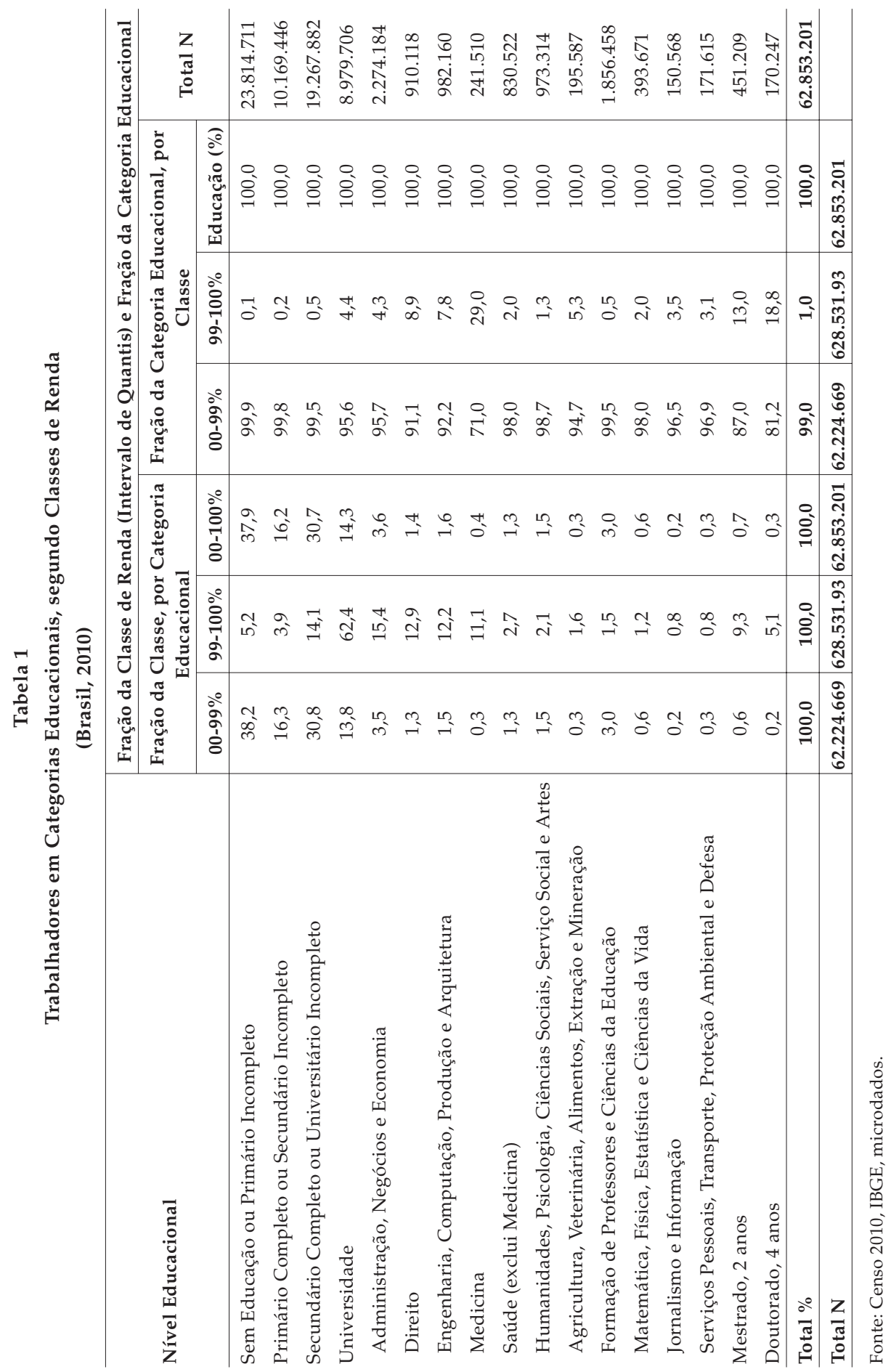

366 DADOS - Revista de Ciências Sociais, Rio de Janeiro, vol. 59, n-2, 2016 
e Economia, ii) Direito, iii) Engenharia, Ciências da Computação, Produção, Construção e Arquitetura e iv) Medicina. Se forem adicionados os dois níveis de pós-graduação, v) Mestrado e vi) Doutorado, as seis categorias agregam $86 \%$ da classe dos ricos. Evidentemente, essa composição poderia estar simplesmente refletindo a composição geral da força de trabalho. No entanto, não é o caso. A fração da força de trabalho total com um diploma universitário nas primeiras quatro categorias, 7,0\% é, na verdade, menor do que a fração em todas as demais categorias, 7,3\%. Possuir um diploma universitário geral de modo algum parece ser suficiente para colocar alguém acima da linha de riqueza. Ao que tudo indica, por trás dos rendimentos mais elevados há algum tipo de educação de elite.

Em boa medida essas categorias refletem o que se observa quando se analisam cursos superiores desagregados (o resultado não é mostrado na Tabela 1). Criamos três listas de dez cursos superiores, uma para os cursos mais comuns na força de trabalho, uma para os mais comuns entre o $1 \%$ mais rico e uma última para os cursos nos quais as chances de estar entre o $1 \%$ mais rico são maiores. Os principais cursos em termos de população total são aqueles relacionados à administração de organizações e da vida civil - Administração, Marketing, Direito e Contabilidade - e ao ensino - Pedagogia, Licenciatura de Professores e Línguas. Em bem menor grau encontram-se cursos relacionados a serviços de saúde - Medicina e Enfermagem. Na lista de dez cursos mais importantes em termos de população não há cursos diretamente relacionados à produção de bens, tais como Engenharia ou Agronomia.

A fração da população total em cada curso reflete-se, em algum grau, na fração da população segundo cursos dentro do $1 \%$ mais rico, embora todos os cursos fortemente relacionados à formação de professores para ensino primário e secundário deixem de constar na lista dos dez mais comuns entre o 1\% mais rico. Os cursos relacionados à administração de organizações e da sociedade persistem, bem como persistem os relacionados aos serviços de saúde, apesar da saída dos cursos de saúde que não a Medicina. Entre os mais comuns no 1\% mais rico passam a constar aqueles diretamente relacionados à produção de bens, com a presença dos cursos de Engenharia. As chances de pertencer ao $1 \%$ mais rico são maiores para aqueles com diplomas em Medicina, Finanças e Seguros (atuária), Engenharia, Serviços de Transporte, e Defesa e Forças Armadas, sendo que os dois últimos possuem um pequeno número de graduados. 


\section{Chances Relativas}

A associação entre educação de elite e posição no topo da distribuição de rendimentos do trabalho é notada quando se observa a distribuição do nível educacional dos trabalhadores, segundo classe de renda na Tabela 1. Quanto maior a educação, maiores são as chances de pertencer ao $1 \%$ mais rico da força de trabalho. Apenas $0,1 \%$ daqueles com menos que educação primária completa pertence à classe dos ricos, mas essa incidência sobe para $4,4 \%$ entre os trabalhadores com diploma universitário e eleva-se para 18,8\% para aqueles com doutorado. Todavia, pertencer ao $1 \%$ mais rico está relacionado não somente ao nível, mas, também, ao tipo de educação recebida. Quando o nível universitário é desagregado, pessoas em certos cursos têm chances muito maiores de pertencer ao $1 \%$ mais rico. Para aquelas pessoas com cursos na área de Ensino as chances de estar no 1\% mais rico são aproximadamente as mesmas daquelas com somente educação secundária; para as que têm um diploma de Medicina, porém, as chances ultrapassam as daquelas com doutorado.

Evidentemente a composição dos diferentes grupos educacionais poderia estar afetando as chances apresentadas na Tabela 1. No entanto, depois de controlar por características individuais, tais como idade, raça e gênero, bem como por segmentação regional do mercado de trabalho, o resultado persiste: possuir educação de elite é muito mais importante do que simplesmente possuir um diploma universitário, como mostra a Tabela 2. De certo modo esse é um resultado esperado, uma vez que nossa definição de curso de elite é dada pela incidência dos cursos no $1 \%$ mais rico. O que chama a atenção, porém, não é a posição, e sim a distância entre os cursos: as chances de estar no $1 \%$ mais rico são várias vezes maiores para trabalhadores com diploma de cursos de elite.

A variação entre níveis educacionais e categorias de cursos é considerável. As razões de chances obtidas por regressão logística na Tabela 2 indicam que as chances que um trabalhador tem de pertencer ao $1 \%$ mais rico crescem substantivamente com a educação universitária. Um trabalhador com educação primária completa tem pouco mais que o dobro das chances de estar no $1 \%$ mais rico que um trabalhador com educação primária incompleta. Esta razão sobe para mais de quatro vezes e meia para trabalhadores com educação secundária completa e 


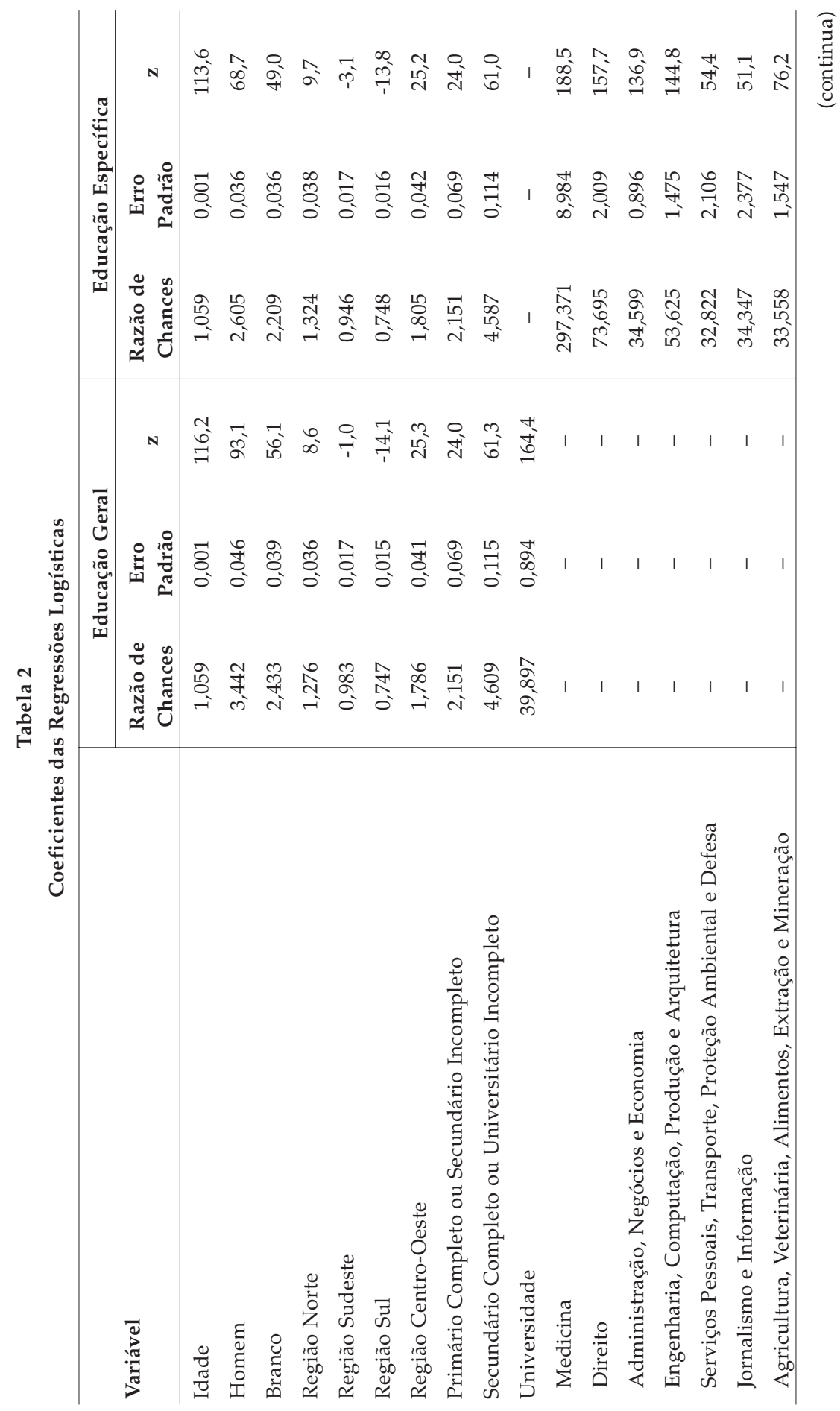


Marcelo Medeiros e Juliana de Castro Galvão

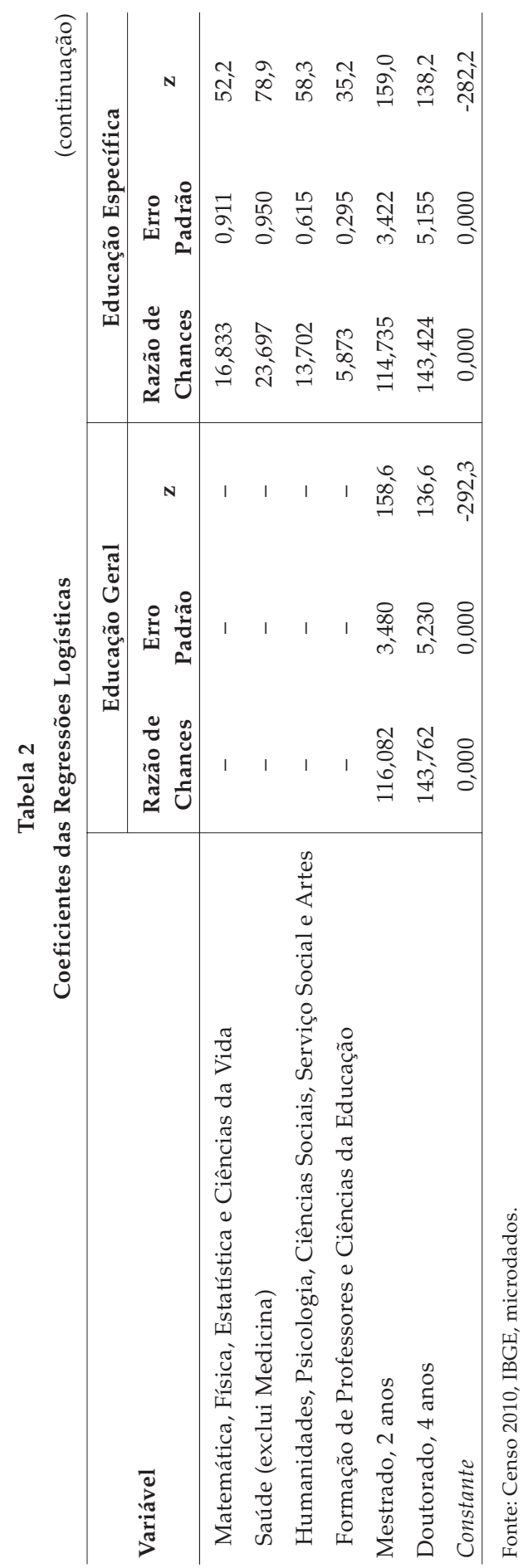


quase 40 vezes mais para aqueles com diploma universitário (sem diferenciar cursos).

Desagregando-se o ensino de graduação por categorias de cursos, observa-se uma desigualdade expressiva nas chances de estar no $1 \%$ mais rico em função do tipo de curso concluído. Os trabalhadores com diplomas na categoria Formação de Professores e Ciências da Educação têm chances quase tão baixas quanto às daqueles com apenas o ensino secundário. Aqueles com diplomas em Humanidades, Psicologia, Ciências Sociais, Serviço Social e Artes - muitos dos quais, na prática, receberam uma educação predominantemente orientada à formação de professores - têm maiores chances, mas encontram-se ainda entre as categorias educacionais inferiores. As chances de estar no $1 \%$ mais rico tornam-se expressivamente maiores para aqueles com treinamento em Engenharia e atividades correlatas (chances quase 54 vezes maiores que as de um trabalhador sem educação primária completa), Direito (74 vezes maiores) e Medicina (297 vezes). Em termos relativos, trabalhadores nas últimas três categorias têm, respectivamente, 9 , 13 e 51 vezes chances maiores de pertencer ao $1 \%$ mais rico que aqueles com diplomas relacionados à Formação de Professores e Ciências da Educação.

\section{Riqueza}

Os resultados apresentados indicam que é a educação de elite, e não simplesmente a educação universitária geral, o que aumenta as chances de alguém ocupar o topo da distribuição de rendimentos do trabalho. Evidentemente, isto se refere aos indivíduos. Nossa preocupação, agora, passa a ser como a educação diferencial pode ser relacionada aos níveis gerais de riqueza. Para isso, analisaremos distribuições hipotéticas de rendimento, simuladas assumindo-se que todos os indivíduos retêm suas características e as respostas do mercado de trabalho a essas características (retornos), exceto educação.

Nosso propósito é examinar o quanto uma educação de elite é essencial para estar no topo da distribuição. Em particular, simulamos o resultado de reduções na educação observada da população, ou seja, o que ocorreria caso os trabalhadores não tivessem mais do que certo nível de educação. Nenhum outro fator, inclusive resíduos, foi alterado nas simulações. Se a educação de elite é essencial, então seria esperada uma redução substancial nas medidas de riqueza após as simulações. 
Gráfico 1

Curvas de Quantis: Rendimentos Observados e Simulados, Reduzindo-se os Retornos à Educação

(Brasil, 2010)

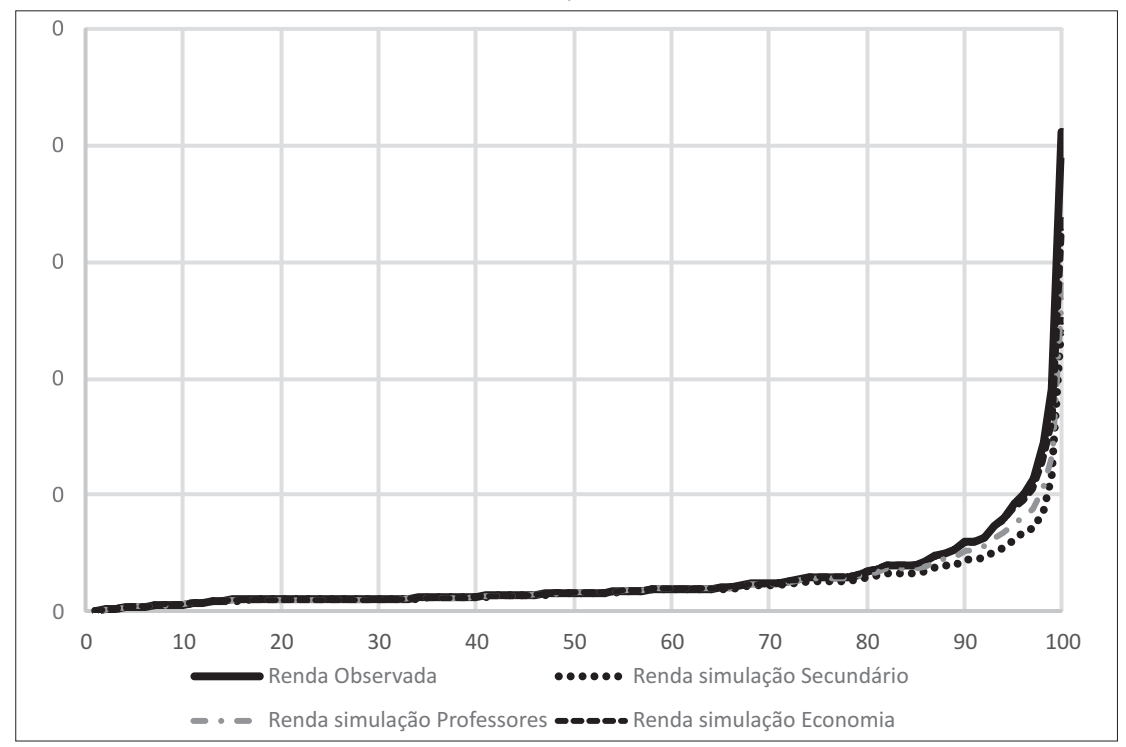

Fonte: Censo 2010, IBGE, microdados.

O Gráfico 1 contém uma curva de quantis ou Parada de Pen dos rendimentos do trabalho. No eixo horizontal foram marcadas as frações de população, de $0 \%$ a $100 \%$ e, no eixo vertical, o rendimento médio recebido por cada centésimo da população. A curva linha contínua representa os rendimentos observados no Censo. As outras curvas simulam o que ocorreria com a distribuição dos rendimentos do trabalho caso as pessoas fossem remuneradas, na média, como se possuíssem um nível de escolaridade diferente daquele que realmente têm. As curvas simulam os cenários em que nenhum trabalhador tem retornos à educação superiores aos de um diploma em Administração, Negócios e Economia, Formação de Professores e Ciências da Educação e educação secundária. Nessas simulações impõe-se um teto à educação, ou seja, o retorno à educação é reduzido somente se o trabalhador possuir um nível educacional superior ao correspondente a cada curva.

Os resultados indicam que sem o efeito diferencial dos cursos de elite os rendimentos mais altos cairiam, mas essa queda não seria substancial. Em média, o rendimento dos ricos cairia $17 \%$ caso o efeito da educação tivesse como teto o nível da categoria Administração, Negócios e Econo- 
mia. Muito mais expressiva seria a mudança causada pelo estabelecimento do teto no nível secundário: rendas cairiam 39\%, em média.

Até mesmo este último resultado deve ser interpretado com cautela. Primeiro, deve-se ter em conta que na simulação do nível secundário estão sendo reduzidos os efeitos da educação e, portanto, os rendimentos, de $62 \%$ dos trabalhadores (Tabela 1). Segundo, ao que parece, o simples fato de os trabalhadores possuírem diplomas universitários não é suficiente para explicar os níveis de riqueza. As simulações sugerem que, em termos de rendimentos, há pouca diferença entre ter apenas educação secundária e um diploma na categoria Formação de Professores e Ciências da Educação.

Apenas uma fração dos indivíduos cairia abaixo da linha de riqueza caso o efeito dos cursos de elite fosse removido. Os trabalhadores caindo abaixo do nível observado do centil $1 \%$ mais rico seriam aqueles próximos a essa linha. Isso fica mais claro quando comparamos linhas de riqueza. Na Tabela 3, a seguir, são apresentadas as variações relativas, em relação aos níveis observados de riqueza, causadas pelas simulações, segundo as medidas de riqueza mais comuns. Em vez de apresentar o valor de cada medida - as quais geralmente não têm um significado substantivo claro - o valor de cada medida observada de riqueza foi padronizado como sendo igual a um e a variação em relação a essa situação inicial calculada depois de cada simulação.

Na Tabela 3, por exemplo, é possível ver que colocar um teto aos retornos à educação equivalente ao retorno da categoria Administração, Negócios e Economia reduz a proporção de ricos (a qual, por definição, é $1 \%$ ) para $0,7 \%$. Estabelecer o teto no nível secundário reduz a proporção de ricos de $1 \%$ para $0,4 \%$ da população, isto é, mesmo depois de removido totalmente o efeito líquido de quaisquer diplomas universitários, $40 \%$ dos ricos observados permaneceriam acima da linha de riqueza.

Medidas que, além da incidência, também consideram a intensidade da riqueza, acabam chegando a conclusões semelhantes. Todas essas medidas caem à metade de seus valores observados quando o teto do retorno educacional é estabelecido no nível da educação secundária. A diferença entre os efeitos de educação secundária e os efeitos de um diploma universitário na categoria Formação de Professores e Ciências da Educação não tem relevância. 
Tabela 3

Variação Relativa nas Medidas de Riqueza

(Brasil 2010)

\begin{tabular}{l|c|c|c|c|c}
\hline Medida & $\begin{array}{c}\text { Renda Observada } \\
\text { (Medida } \\
\text { Absoluta) }\end{array}$ & $\begin{array}{c}\text { Renda } \\
\text { Observada }\end{array}$ & $\begin{array}{c}\text { Renda } \\
\text { Simulação } \\
\text { Economia }\end{array}$ & $\begin{array}{c}\text { Renda } \\
\text { Simulação } \\
\text { Professores }\end{array}$ & $\begin{array}{c}\text { Renda } \\
\text { Simulação } \\
\text { Secundário }\end{array}$ \\
\hline HCR & 0,0100 & 1,0 & 0,7 & 0,5 & 0,4 \\
FGTT1(0,1) & 0,0085 & 1,0 & 0,7 & 0,5 & 0,4 \\
FGTT1(0,3) & 0,0066 & 1,0 & 0,7 & 0,5 & 0,4 \\
FGTT1(1) & 0,0032 & 1,0 & 0,7 & 0,5 & 0,4 \\
Cha(0,1) & 0,0004 & 1,0 & 0,7 & 0,5 & 0,4 \\
Cha(0,3) & 0,0012 & 1,0 & 0,7 & 0,5 & 0,4 \\
Cha(1) & 0,0032 & 1,0 & 0,7 & 0,5 & 0,4 \\
Cha(3) & 0,0060 & 1,0 & 0,7 & 0,4 & 0,4 \\
Cha(10) & 0,0085 & 1,0 & 0,7 & 0,4 & 0,4 \\
FGTT2(1) & 0,0087 & 1,0 & 0,7 & 0,5 & 0,5 \\
FGTT2(2) & 0,0772 & 1,0 & 0,7 & 0,5 & 0,5 \\
\hline
\end{tabular}

Fonte: Censo 2010, IBGE, microdados.

Nota: linha de riqueza de R\$ 11.000 (topo 1\%), HCR: incidência, FGTT1(a): índice FGT de riqueza cônvaco (axioma T1), Cha(b): índice Chakravaty côncavo (axioma T1), FGTT2(a): convexo (axioma T2) (Peichl, Schaefer e Scheicher, 2010).

Aparentemente há fatores não observados que tendem a ser muito mais importantes para explicar a diferença entre os ricos e o resto. Poderia estar entre estes fatores a qualidade da educação, em particular a qualidade da educação superior? Esta é uma possibilidade pouco plausível. Há, indiscutivelmente, diferenças entre universidades, mas antes de assumi-las como uma explicação relevante é preciso ter em mente alguns fatos. Primeiro, há no Brasil, como em outros lugares, seleção de clientela: algumas universidades têm melhor desempenho não tanto porque são melhores em termos de ensino, mas, principalmente, porque atraem os melhores alunos - devido à seleção de clientela, uma pequena vantagem em qualidade pode resultar em uma imensa diferença de desempenho. Segundo, a qualidade dos cursos de elite é direta ou indiretamente controlada por uma série de instituições, e a parte técnica desses cursos é razoavelmente padronizada Direito, Medicina e Engenharia, cursos dominantes no 1\% mais rico, são exemplos. Diferenças em relação à qualidade existem, mas é difícil conceber um grande número de cursos de elite cuja qualidade é várias vezes superior a cursos similares em outras universidades, a ponto de 
justificar as grandes diferenças de escala evidenciadas nas Paradas de Pen. Levando tudo isso em consideração, parece adequado descartar a hipótese da qualidade até que dados melhores que os nossos eventualmente apontem evidências na direção contrária.

As mudanças nas Paradas de Pen e nas medidas de riqueza nos fazem crer que, embora a maioria do $1 \%$ mais rico tenha um diploma universitário, o efeito geral de se ter educação superior não é uma razão importante para colocar essas pessoas no topo da distribuição. Ao que parece, apenas a educação de elite é um fator explicativo forte da riqueza. Não podemos perder de vista que, mesmo após a supressão desse efeito, uma grande parte do $1 \%$ mais rico não sofre mobilidade descendente a ponto de abandonar a classe do $1 \%$ mais rico.

\section{CONCLUSÕES}

A educação pode ser importante para explicar a desigualdade total, mas não há evidências de que a educação de massa seja um dos fatores mais relevantes para explicar as diferenças entre os ricos e o resto da população no Brasil. Obviamente, esta é uma questão de interpretação, mas acreditamos que a maneira apropriada de contemplar os resultados obtidos é concluir que nem mesmo a educação de elite pode ser tomada como um dos determinantes principais dos níveis atuais de riqueza. A educação de elite seguramente diferencia algumas pessoas e provavelmente é um determinante importante da riqueza de alguns trabalhadores no 1\% mais rico, mas uma grande parte dessas pessoas seria rica mesmo sem a contribuição líquida da educação para seus rendimentos. Portanto, não se deve assumir que os ricos são ricos, predominantemente, porque são mais educados, mesmo quando consideramos indicadores importantes como o tipo de formação de nível superior.

Os ricos concentram uma fração completamente desproporcional da renda no país. Por essa razão, sua contribuição para a desigualdade é muito grande. Pelo fato de os ricos não serem ricos apenas (ou principalmente) porque têm mais e melhor educação, deve haver uma parcela grande da desigualdade que não poderá ser reduzida por políticas educacionais, mesmo políticas capazes de elevar o nível educacional dos trabalhadores a ponto de massificar aquilo que hoje é educação de elite. Em outras palavras, melhorar o nível educacional da população pode ser condição necessária, mas provavelmente não será suficiente 
para se atingir níveis razoavelmente baixos de desigualdade nas próximas décadas. $\mathrm{O}$ quanto, exatamente, precisa ser estimado por estudos futuros.

(Recebido para publicação em abril de 2015)

(Reapresentado em dezembro de 2015)

(Aprovado para publicação em fevereiro de 2016) 


\section{REFERÊNCIAS BIBLIOGRÁFICAS}

ALBUQUERQUE, Paula Medeiros. (1994), Um Estudo da População de Altos Rendimentos no Brasil nos Anos Recentes. Rio de Janeiro, Ipea.

ALVAREDO, Facundo. (2010), "The Rich in Argentina over the Twentieth Century: 1932-2004", in A. B. Atkinson; T. Piketty (eds.), Top Incomes: A Global Perspective. Oxford, Oxford University Press, pp. 253-298.

ATKINSON, Anthony B. (2007), “Measuring Top Incomes: Methodological Issues”, in A. B. Atkinson; T. Piketty (eds.), Top Incomes over the Twentieth Century: A Contrast between Continental European and English-Speaking Countries. Oxford, Oxford University Press, pp. 18-42.

; PIKETTY, Thomas; SAEZ, Emmanuel. (2011), “Top Incomes in the Long Run of History". Journal of Economic Literature, vol. 49, no 1, pp. 3-71.

BAKIJA, Jon; COLE, Adam; HEIM, Bradley T. (2010), “Jobs and Income Growth of Top Earners and the Causes of Changing Income Inequality: Evidence from US Tax Return Data". Working Papers. Department of Economics, Williams College.

BREEN, Richard; JONSSON, John O. (2005), “Inequality of Opportunity in Comparative Perspective: Recent Research on Educational Attainment and Social Mobility". Annual Review of Sociology, vol. 31, pp. 223-243.

BROOM, Leonard; SHAY, William. (2000), “Discontinuities in the Distribution of Great Wealth: Sectoral Forces Old and New". Economics Working Paper Archive. Levy Economics Institute.

CANTERBERY, E. Ray; NOSARI, E. Joe. (1985), “The Forbes Four Hundred: The Determinants of Super-Wealth". Southern Economic Journal, vol. 51, no 4, pp. 1073-1083.

ERIKSON, Robert; GOLDTHORPE, John H. (2002), “Intergenerational Inequality: A Sociological Perspective". Journal of Economic Perspectives, vol. 16, no 3, pp. 31-44.

FEENBERG, Daniel R.; POTERBA, James M. (2000), "The Income and Tax Share of Very High-Income Households, 1960-1995". The American Economic Review, vol. 90, no 2, pp. 264-270.

FERREIRA, Marcelo C. (2001), “Permeável, ma non Troppo? A Mobilidade Social em Setores de Elite, Brasil - 1996". Revista Brasileira de Ciências Sociais, vol. 16, no 47, pp. 141-160.

FINNIE, Ross; IRVINE, Ian. (2006), “Mobility and Gender at the Top Tail of the Earnings Distribution". Economic and Social Review, vol. 37, no 2, pp. 149-173.

FORTIN, Nicole et al. (2012), “Canadian Inequality: Recent Developments and Policy Options". Canadian Public Policy, vol. 38, no 2, pp. 121-145.

GODECHOT, Olivier. (2012), "Is Finance Responsible for the Rise in Wage Inequality in France?". Socio-Economic Review, vol. 10, no 3, pp. 447-470.

HURD, Michael D.; MUNDACA, B. Gabriela. (1989), "The Importance of Gifts and Inheritances among the Affluent", in R. E. Lipsey; H. S. Tice (eds.), The Measurement of Saving, Investment, and Wealth. Chicago, University of Chicago Press, pp. 737-764. 
INHABER, Herbert; CARROLL, Sidney L. (1992), How Rich Is too Rich? Income and Wealth in America. New York, Praeger.

KAPLAN, Steven N.; RAUH, Joshua. (2010), “Wall Street and Main Street: What Contributes to the Rise in the Highest Incomes?". Review of Financial Studies, vol. 23, no 3, pp. 1004-1050.

KEISTER, Lisa A. (2014), "The One Percent". Annual Review of Sociology, vol. 40, pp. 347-367.

; LEE, Hang Y. (2014), “The One Percent Top Incomes and Wealth in Sociological Research". Social Currents, vol. 1, no 1, pp. 13-24.

LANDAIS, Camille. (2008), Top Incomes in France: Booming Inequalities?. Paris School of Economics, Mimeo, Paris.

LEIGH, Andrew; VAN der ENG, Pierre. (2009), “Inequality in Indonesia: What Can we Learn from Top Incomes?" Journal of Public Economics, vol. 93, no 1, pp. 209-212.

LUNDBERG, Ferdinand. (1969), The Rich and the Super-Rich: A Study in the Power of Money Today. New York, Bantam Books.

MEDEIROS, Marcelo. (2005), O que Faz os Ricos Ricos: O Outro Lado da Desigualdade Brasileira. São Paulo, Editora Hucitec/Anpocs.

; SOUZA, Pedro Herculano G. F.; CASTRO, Fábio A. (2015a), “O Topo da Distribuição de Renda no Brasil: Primeiras Estimativas com Dados Tributários e Comparação com Pesquisas Domiciliares, 2006-2012". DADOS - Revista de Ciências Sociais, vol. 58, no 1, pp. 7-36.

. (2015b), "The Stability of Income Inequality in Brazil, 2006-2012: An Estimate Using Income Tax Data and Household Surveys". Ciência \& Saúde Coletiva, vol. 20, no 4, pp. 971-986.

PARKER, Jonathan A.; VISSING-JORGENSEN, Annette. (2010), “The Increase in Income Cyclicality of High-Income Households and Its Relation to the Rise in Top Income Shares". Brookings Papers on Economic Activity, pp. 1-70.

PEICHL, Andreas; SCHAEFER, Thilo; SCHEICHER, Christoph. (2010), "Measuring Richness and Poverty: A Micro Data Application to Europe and Germany". Review of Income and Wealth, vol. 56, no 3, pp. 597-619.

PIKETTY, Thomas. (2000), "Theories of Persistent Inequality and Intergenerational Mobility", in A. B. Atkinson; F. Bourguignon (eds.), Handbook of Income Distribution. Oxford/New York, Elsevier, pp. 429-476.

; SAEZ, Emmanuel. (2013), “Top Incomes and the Great Recession: Recent Evolutions and Policy Implications". IMF Economic Review, vol. 61, no 3, pp. 456-478.

RUSSELL, Thomas. (1979), “The Share of Top Wealth Holders: The Life Cycle, Inheritance and Efficient Markets". Annales de L'Inséé, no 33/34, pp. 159-180.

SAEZ, Emmanuel. (2006), "Income and Wealth Concentration in Historical and International Perspective", in A. J. Auerbach; D. Card; J. M. Quigley (eds.), Public Policy and the Income Distribution. New York, Russell Sage Foundation, pp. 221-258. 
;VEALL, Michael R. (2005), “The Evolution of High Incomes in Northern America: Lessons from Canadian Evidence". The American Economic Review, vol. 95, no 3, pp. 831-849.

SANHUEZA, Claudia; MAYER, Ricardo. (2011), “Top Incomes in Chile Using 50 Years of Household Surveys: 1957-2007". Estudios de Economía, vol. 38, no 1, pp. 169-193.

SOROKIN, Pitirim. (1925), "American Millionaires and Multi-Millionaires: A Comparative Statistical Study". Journal of Social Forces, vol. 3, no 4, pp. 627-640.

SPILERMAN, Seymour. (2000), "Wealth and Stratification Processes". Annual Review of Sociology, vol. 26, no 1, pp. 497-524.

STILWELL, Frank; JORDAN, Kirrily. (2007), Who Gets What?: Analysing Economic Inequality in Australia. Cambridge, Cambridge University Press.

TICKAMYER, Ann R. (1981), “Wealth and Power: A Comparison of Men and Women in the Property Elite". Social Forces, vol. 60, no 2, pp. 463-481.

TORCHE, Florencia. (2014), "Intergenerational Mobility: The Latin American Case". Annual Review of Sociology, vol. 40, pp. 619-642.

; COSTA-RIBEIRO, Carlos. (2012), "Parental Wealth and Children's Outcomes over the Life-course in Brazil: A Propensity Score Matching Analysis". Research in Social Stratification and Mobility, vol. 30, no 1, pp. 79-96.

TORCHE, Florencia; SPILERMAN, Seymour. (2008), “Household Wealth in Latin America", in J. B. Davies (ed.), Personal Wealth from a Global Perspective. Oxford, Oxford University Press.

. (2009), "Intergenerational Influences of Wealth in Mexico". Latin American Research Review, vol. 44, no 3, pp. 75-101.

VÉLEZ, Juliana L. (2012), Income and Wealth at the Top in Colombia: An Exploration of Tax Records 1993-2010. Dissertação (Mestrado em Políticas Públicas). Paris School of Economics, Paris.

WOLFF, Edward N. (2000), "Who Are the Rich? A Demographic Profile of High-income and High-wealth Americans", in J. Slemrod (ed.), Does Atlas Shrug? The Economic Consequences of Taxing the Rich. Cambridge, MA, Harvard University Press, pp. 74-113.

(2002), "Inheritances and Wealth Inequality, 1989-1998". The American Economic Review, vol. 92, no 2, pp. 260-264. 


\section{RESUMO}

\section{Educação e Rendimentos dos Ricos no Brasil}

$\mathrm{O}$ artigo examina em que medida a educação pode ser considerada um dos principais determinantes da riqueza no Brasil. Utilizando os dados de formação universitária específica da Amostra do Censo 2010, o foco restringe-se ao $1 \%$ mais rico da distribuição de rendimentos do trabalho. A principal conclusão é que a educação pode ser importante para explicar a desigualdade total, mas não há evidências de que a educação de massa seja um dos fatores mais relevantes para explicar as diferenças entre os ricos e o resto da população brasileira. Nem mesmo a educação de elite pode ser tomada como um dos principais determinantes dos níveis atuais de riqueza. Há, portanto, uma parte importante da desigualdade total que não será reduzida por políticas educacionais.

Palavras-chave: estratificação social; desigualdade de renda; ricos; educação; desigualdade salarial

\section{ABSTRACT \\ Education and Income of the Rich in Brazil}

This article examines the extent to which education can be considered one of the main determinants of wealth in Brazil. Using date pertaining to the specific university education from the 2010 Census Sample, it focuses on the $1 \%$ richest within the distribution of income. The main conclusion is that education may be important in explaining total inequality, but there is no evidence that mass education is one of the most relevant factors in explaining the differences between the rich and the rest of the Brazilian population. Not even elite education can be considered a main determinant of the current levels of wealth. Therefore a significant portion of total inequality cannot be reduced by educational policy.

Keywords: social stratification; income inequality; the rich; education; salary inequality 


\section{RÉSUMÉ}

\section{Éducation et Revenus des Riches au Brésil}

Cet article examine dans quelle mesure l'éducation peut être considérée comme l'un des principaux facteurs déterminants de la richesse au Brésil. À partir des données de formation universitaire spécifique du recensement de 2010, nous avons restreint notre échantillon aux 1\% les plus riches de la distribution des revenus du travail. La principale conclusion est que l'éducation peut être importante pour expliquer globalement les inégalités, mais rien ne prouve que l'éducation de masse constitue l'un des facteurs les plus pertinents pour expliquer les différences entre les riches et le reste de la population brésilienne. L'éducation de l'élite ne peut pas non plus être considérée comme l'un des principaux déterminants des niveaux actuels de richesse. Il existe donc une part importante des inégalités dans leur ensemble qui ne saurait être réduite par les seules politiques éducatives.

Mots-clés: stratification sociale; inégalités de revenus; riches; éducation; inégalités salariales

\section{RESUMEN}

\section{Educación y Rendimientos de los Ricos en Brasil}

El artículo examina en qué medida la educación puede ser considerada uno de los principales determinantes de la riqueza en Brasil. Valiéndose de los datos de formación universitaria específica del Censo de 2010, el foco se restringe al $1 \%$ más rico de la distribución de rendimientos de trabajo. La principal conclusión es que la educación puede ser importante para explicar la desigualdad total, pero no hay evidencias de que la educación en masa sea uno de los factores más relevantes para explicar las diferencias entre los ricos y el restante de la población brasileña. Ni siquiera la educación de elite puede ser tomada como uno de los principales determinantes de los actuales niveles de pobreza. Por lo tanto, hay una parte significativa de la desigualdad total que no será reducida por políticas educativas.

Palabras clave: estratificación social; desigualdad de renta; ricos; educación; desigualdad salarial 